\title{
An Adaptive Compliant Multi-finger Approach-To-Grasp Strategy for Objects with Position Uncertainties
}

\author{
Zhaopeng Chen, Thomas Wimböck, Maximo A. Roa, Benedikt Pleintinger, Miguel Neves \\ Christian Ott, Christoph Borst, and Neal Y. Lii
}

\begin{abstract}
This paper presents an adaptive and compliant approach-to-grasp strategy for multi-finger robotic hands, to improve the performance of autonomous grasping when encountering object position uncertainties. With the proposed approach-to-grasp strategy, the first robot finger to experience unexpected impact would pause its movement in a compliant manner, and remains in contact with the object to minimize the unplanned motion of the target object. At the same time, the remainder of the fingers continuously, adaptively move toward re-adjusted grasping positions with respect to the first finger in contact with the object, without the need for on-line replanning or re-grasping. An adaptive grasp control strategy based on spatial virtual spring framework is proposed to achieve local (e.g. not resorting to the robotic arm) in-hand adjustments of the fingers not yet in contact. As such, these fingers can be adaptively driven to the adjusted desired position to accomplish the grasp. Experimental results demonstrate that significantly larger position errors with respect to the hand workspace can be accommodated with the proposed adaptive compliant grasp control strategy. As much as $391 \%$ increase in position error area coverage has been achieved. Finally, beyond the quantitative analysis, additional observations during the extensive experiment trials are discussed qualitatively, to help examine several open issues, and further understand the approach-to-grasp phases of the robot hand tasks.
\end{abstract}

\section{INTRODUCTION}

Autonomous grasping with multi-finger dexterous robot hands have gained much of attention in recent decades. The larger number of fingers intrinsically offers more grasp robustness than that of the well-studied two-jaw grippers, and provide further in-hand manipulation ability. On the other hand, with a multi-finger grasp, thanks to the increased number of contact points with the object, the object in-hand pose can be more effectively estimated to help facilitate improved execution of the follow-up task such as object manipulation.

Typically, grasp selection is made prior to grasp execution, based on known object model or information acquired for grasp planning [1]. Completion of the grasp would be sufficient for most two-jaw grippers and under-actuated hands, where grasping of the object is usually the final goal for the robot. However, for a dexterous robot hand, the grasp of the target object is not the end of the task, but the beginning to

All the authors are with Robotics and Mechatronics Center, German Aerospace Center, DLR, 82234 Wessling, Germany. zhaopeng. chendalr.de further tasks such as transporting or manipulating the object. Errors in the predicted object position and robot control can manifest into failed grasps, or failure and/or difficulty in executing object tasks further down the task chain. In these scenarios the objects are not in the expected location for the robot, or the end-effector of the robot is not in the expected configuration as the robot is commanded. As a result, the actual grasping points and robot hand/finger configurations cannot be obtained as planned. Furthermore, unexpected contacts or collisions caused by the uncertainties during the grasping task execution would further hinder the quality of the following grasps.

In our previous work on in-hand grasping and manipulation, we observed a reduction in performance with the presence of object position errors [2]. In recent years, contact detection, object estimation. Related reactive grasp control strategies have been investigated to help improve grasp execution. An in-hand object location estimation method based on Particle Filter was proposed in [3]. However, post-grasp phase object localization problem is tackled in this work, rather than grasp execution phase. A robust parallel force and position control is proposed by Takahashi et al.[4]. The robust adaptive grasp control is achieved through tactile-based force and position control, and promising results are yielded. However, objects grasping with unknown stiffnesses and shapes are addressed in this work, rather than object position errors. Hsiao et al.[5] introduced a contact-reactive grasping strategy for objects with partially known information based on tactile sensors. The experiments were conducted with the PR2 personal robot with parallel two-jaw grippers mounted on both arms of the robot. Similar work was investigated in [6]. However, multi-finger dexterous grasp approach method is not tackled in this work. Furthermore, the contact forces between the robot fingers and the object can increase the uncertainties as a result of the random motion of the object, on which the object location detection for the reactive grasp strategy is based.

In order to achieve a grasp approach strategy, including contact detection, reactive control during contact, and following adaptive grasp approach control, impedance control framework is utilized in this paper, which was introduced by Hogan [7]. The virtual spatial spring concept is adopted to construct the adaptive compliant grasp control scheme, 
which has been introduced by Stramigioli [8]. Wimböck et al. demonstrated the first application of the virtual spatial spring system into multi-finger manipulation, and experiments are carried out on the 4-finger dexterous robotic hand DLR II [9]. Fasse et al. [10] introduced a object level impedance controller based on the virtual spatial spring. Khatib [11] analyzed the inertial properties of the object level manipulation. However, in the works mentioned here, spatial virtual spring based impedance controllers were mainly designed for objects manipulation in post-grasp phase. In this paper, the impedance control and spatial virtual spring concept would be utilized to achieve adaptive compliant control in the approach-to-grasp phase.

Passive compliance has been widely utilized in underactuated robotic hands to achieve adaptively grasping for objects with uncertainties [12]. The passive behavior of the fingers with adaptive mechanisms allows them to wrap around the object without the need of sensing or control, thus obtaining stable power grasps [13][14]. However, these approaches tend to have limited or no in-hand manipulation capabilities, as they are not intended to control individual degrees of freedom in the fingers. The un-modeled information, such as object location errors and un-expected contacts cannot be detected, as a result of the lack of sensory feedback. Recently, the possibility of obtaining basic manipulation capabilities with under-actuated hands has been explored [15][16]. In contrast to these works, the proposed framework enables a multi-finger hand to adapt the grasp approach according to the real environment, thus creating a system robust to pose uncertainties in the object location with respect to the hand, while still retaining the capability of in-hand manipulation that fully actuated multi-finger hands provide.

This paper is organized as follows: Section II describes the adaptive compliant approach-to-grasp strategy. Section III describes the contact detection and reactive compliant control during contact. Section IV presents the adaptive multi-finger compliant grasp controller based on the virtual spatial spring, including 2-finger case and multi-finger case; quantitative results and analysis on our experiments are presented in Section V. This is followed by more qualitative observations and pondering in Section VI. Finally, the conclusions of this work are presented in Section VII.

\section{The Adaptive Compliant Approach-to-Grasp STRATEGY}

Uncertainty of the target object's location, is one of the main obstacles for autonomous grasp task execution with robotic hands and grippers. In order to address the position uncertainty problem in an adaptive and compliant manner, several criteria should be addressed during the grasp approach strategy design:

- Position errors of the target object should be detected by the robot finger in real-time;
- Unexpected motion of the object during the impact should be minimized;

- The robot fingers should be locally (e.g. in-hand) adjusted to achieve stable grasp.

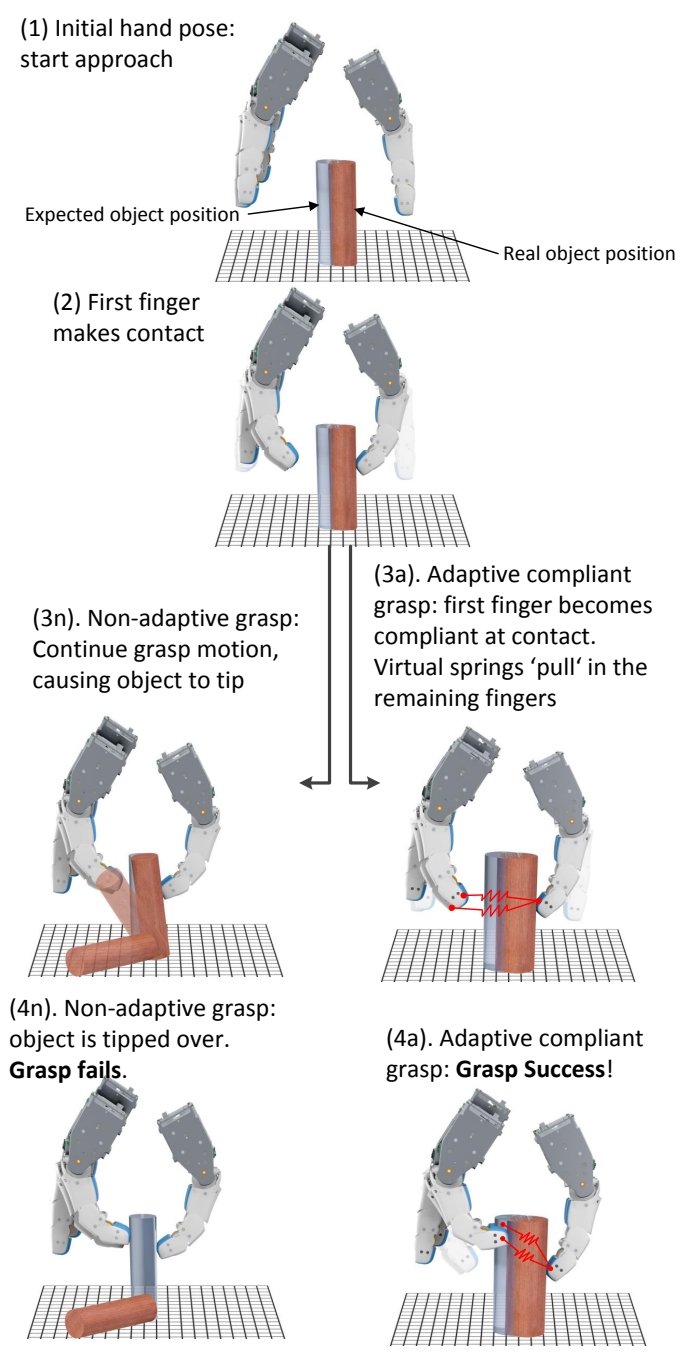

Fig. 1. The non-adaptive and adaptive concepts for approach-to- grasp strategy. As the hand starts its approach toward the target object ((1)-(2)), one finger would make a first contact unexpectedly due to the object position error ((2)-(3)). In the non-adaptive grasp approach ((3n)-(4n)), all fingers continue to move toward the expected object position. This can cause the object pose and position to change. We illustrate the example of the object tipping here, which can result in the object falling over in some cases, which in turn causes a grasp failure (4n). Our proposed adaptive compliant grasp approach, on the other hand, would keep the first-contact finger in compliant contact with the object (3a), while using virtual springs to draw the other fingers to the adjusted desired position. The remaining fingers would then move in until every finger makes contact with the object to complete the grasp (4a).

With these criteria, we propose an adaptive and compliant approach-to-grasp strategy for robotic hands, which address the hand's approach toward the object, up until the state of a stable grasp. This strategy, shown in Fig. 1, consists of several sequential steps: 
- When unexpected contact occurs (the joint torque sensor reaches the designed threshold), displacement of the target object is detected by the joint torque sensor integrated in the robot finger

- The reactive compliant control strategy should be employed to stop the finger motion compliantly to reduce the impact force, which in turn minimizes the unplanned motion of the object

- Once the contact is detected, fingers without contact is controlled toward a locally adjusted grasp position, through an compliant grasp approach control strategy based on spatial virtual spring concept, until the target object is stably grasped.

This study's scope is limited to local in-hand adjustment of the fingers in this paper. Vision or tactile feedback are not utilized, and no on-line planner or re-grasp behaviors were required here to complete the grasp execution. In cases where position errors of the target object are beyond the workspace of the robotic hand (when re-grasp is required), the proposed adaptive compliant grasp strategy can be easily extended together with motion adjustment of the robot arm to a achieve stable grasp. Re-grasp strategies, such as [5] and [6], can be employed. The compliant control during the contact, and the compliant grasp approach control based on spatial virtual spring are discussed in more detail in the following sections.

\section{Contact Detection And Compliant Control STRATEGY DURING CONTACT}

A multi-sensory dexterous hand's function is not limited to that of a grasp and manipulation endeffector. The position errors of the object location should also be detected by the robot fingers so that the immediately following adaptive grasp execution sequence can be performed adequately.

The experiments in this study are carried out with a modified robot hand based on the dexterous multi-sensory robot hand DLR/HIT II, mounted on a 7-DoF Kuka Light Weight Robot arm [17][18]. The hand consists of five identical modular 3-DoF fingers. Each DoF is active and integrated with torque and positions sensors. The torque sensors integrated in joints of the hand are utilized to detect unexpected contact between the finger and the object. The available sensitivity of the contact detection is mainly determined by noise of the joint toque sensor, which is less than $0.01 \mathrm{~N} \cdot \mathrm{m}$.

During the approach phase, a joint-torque based Cartesian level impedance controller is utilized to drive the robot finger joints [19]. Once the first unexpected contact is detected, the finger remains in contact with the object in a compliant manner, so that the unplanned motion of the object is minimized. The compliant behavior of the robot finger during contact is realized as:

- $q_{d}(t)=q(t)\left(t \geq t_{c}\right)$, where $t_{c}$ represents the time instant of the contact.
- $k_{p}=0\left(t \geq t_{c}\right)$, where $k_{p}$ indicates the stiffness of the joint impedance controller.

\section{AdAPTIVE COMPLiant APPROACH-TO-GRASP BASEd on The SPatial VirTual SPRing CONCEPT}

\section{A. The Multi-finger Compliant Grasp Approach Strategy}

Due to the identical modular design of the DLR-HIT II robot fingers, an n-finger dexterous robot hand can be constructed for different tasks. In order to achieve adaptive compliant grasp approach, a multi-finger spatial virtual spring frame is proposed in this paper, as shown in Fig. 2. The spatial virtual springs are attached between the thumb and the remaining fingers on the hand. In order to obtain usable distances between the fingers while grasping, additional collision-avoidance virtual springs are attached to different adjacent fingers. These are further elaborated in the next section. A closed form of spatial virtual spring frame is therefore established on a multi-finger robotic hand. When any of the fingers experiences an unexpected contact with the object, the remaining fingers would be "pulled" to the adjusted grasp position by the virtual springs. The rest length of the virtual springs should be chosen as the same as the distance between the fingers while they are in their original desired position, which is defined during the off-line grasp selection phase. In this way, the object is "caged" in the closed form spatial virtual spring frame. The multi-finger virtual spring frame proposed in this paper can be applied to n-finger robot hand configuration with $n \geq 2$. It is assumed in this paper that only the fingertips are in contact with the object while grasping, and the point contact with friction (PCWF) model is taken for the remainder of this paper.

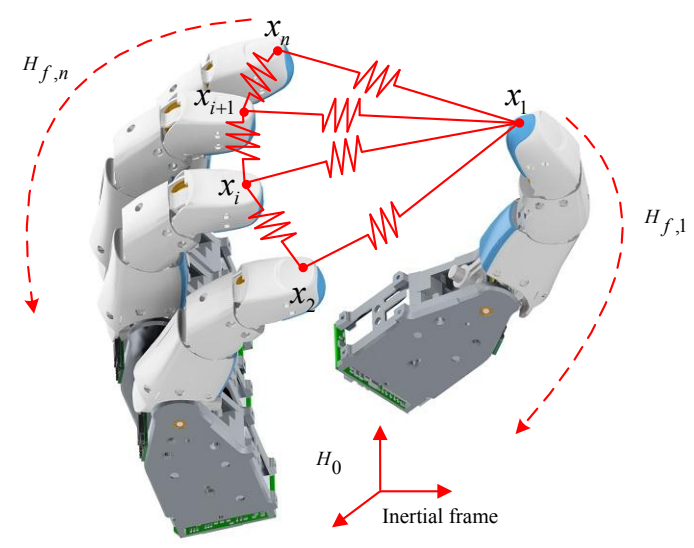

Fig. 2. The spatial virtual spring framework with self-collision avoidance between different fingers during the approaching phase

The spatial virtual spring framework employs $2 n-3$ spatial springs, including the self-collision avoidance virtual spring. Each virtual spring between the thumb and one of the other fingers involved in the grasp task is based on the fingertip Cartesian positions of the two robot fingers, shown in Fig. 
2. The compliant grasp approach control law, based on the virtual spatial spring between the thumb and one of the other fingers can be written as:

$$
\boldsymbol{\tau}_{a d a}=-\frac{\partial V_{1 i}(\boldsymbol{\theta})}{\partial \boldsymbol{\theta}^{T}}-\boldsymbol{D}_{1 i}(\boldsymbol{\theta}) \dot{\boldsymbol{\theta}}+g(\boldsymbol{\theta})
$$

where the actuator torque vector, $\tau_{a d a}$, is considered as the control input. $V_{1 i}$ is the energy stored in the virtual spatial spring. $\boldsymbol{\theta}$ indicates the vector of the joint angle. $D_{1 i}(\boldsymbol{\theta})$ represents the damping term with respect to the joint space, which is mapped from the damping force along the virtual spring direction. $g(\boldsymbol{\theta})$ is the gravity term. Based on the passivity control theory, the energy store function of the virtual spatial spring can be chosen as:

$$
V_{1 i}=\frac{1}{2} K_{1 i}\left(\left\|\Delta \boldsymbol{x}_{1 i}\right\|-l_{o}\right)^{2}
$$

where $\boldsymbol{\Delta} \boldsymbol{x}_{1 i}=\left\|\boldsymbol{x}_{1}-\boldsymbol{x}_{i}\right\|$ represents the distance between the center points of the thumb and one of the remaining fingers. $l_{o}$ indicates the rest length of the virtual spatial spring. $K_{1 i}$ indicates the stiffness parameter of the virtual spring. $x_{1}$ and $x_{i}$ are the Cartesian position of the thumb and one of the remaining fingers. The adaptive grasp controller can be derived from $V_{1 i}$ together with an appropriately designed damping term. The time derivation of the eq. (2) can be written as:

$$
\begin{aligned}
\dot{V}_{1 i} & =-K_{1 i}\left(\boldsymbol{\Delta} \boldsymbol{x}_{1 i}-l_{o}\right)\left\|\boldsymbol{\Delta} \dot{\boldsymbol{x}}_{1 i}\right\| \\
\boldsymbol{F}_{1 i} & =-K_{1 i}\left(\boldsymbol{\Delta} \boldsymbol{x}_{1 i}-l_{o}\right)
\end{aligned}
$$

where $F_{1 i}$ represents the control law of the adaptive grasp controller with respect to Cartesian space.

Considering that $\left\|\Delta x_{1 i}\right\|=\left(\Delta x_{1 i}^{T} \cdot \Delta x_{1 i}\right)^{\frac{1}{2}}$, eq. (3) can be rewritten as:

$$
\begin{aligned}
\frac{\partial V_{1 i}=}{\partial \boldsymbol{\theta}^{T}}= & -\boldsymbol{K}_{1 i}\left(\left\|\boldsymbol{\Delta} \boldsymbol{x}_{1 i}\right\|-l_{o}\right) \cdot \\
& \frac{\boldsymbol{\Delta} \boldsymbol{x}_{1 i}}{\left\|\boldsymbol{\Delta} \boldsymbol{x}_{1 i}\right\|} \frac{\partial \boldsymbol{\Delta} \boldsymbol{x}_{1 i}}{\partial \boldsymbol{x}_{1 i}^{T}}\left(\boldsymbol{x}_{1}^{1}, \boldsymbol{x}_{i}^{i}\right)\left[\begin{array}{cc}
\boldsymbol{J}_{1} & 0 \\
0 & \boldsymbol{J}_{i}
\end{array}\right]
\end{aligned}
$$

with:

$$
\frac{\partial \boldsymbol{\Delta} \boldsymbol{x}_{1 i}}{\partial \boldsymbol{x}_{1 i}^{T}}=\left(\begin{array}{cc}
\boldsymbol{I}_{3 \times 3} & -\boldsymbol{I}_{3 \times 3}
\end{array}\right)
$$

where $\boldsymbol{J}_{1}$ and $\boldsymbol{J}_{i}$ represent the Jacobian matrices mapping coordinates $\boldsymbol{H}_{1}$ and $\boldsymbol{H}_{i}$ into the joint space of the fingers, respectively. The control law derived from the energy storage function of the virtual spatial spring can be mapped into joint space of the fingers with eq. (5). Therefore, the control law with respect to the finger joint space can be expressed as:

$$
\begin{aligned}
\boldsymbol{\tau}_{1 i} & =\boldsymbol{J}_{1 i}^{T} \boldsymbol{F}_{1 i} \\
\boldsymbol{J}_{1 i} & =\frac{\boldsymbol{\Delta} \boldsymbol{x}_{1 i}}{\left\|\boldsymbol{\Delta} \boldsymbol{x}_{1 i}\right\|} \frac{\partial \boldsymbol{\Delta} \boldsymbol{x}_{1 i}}{\partial \boldsymbol{x}_{1 i}^{T}}\left(\boldsymbol{x}_{1}^{1}, \boldsymbol{x}_{1}^{1}\right)\left[\begin{array}{cc}
\boldsymbol{J}_{1} & 0 \\
0 & \boldsymbol{J}_{i}
\end{array}\right]
\end{aligned}
$$

$J_{1 i}$ represents the general Jacobian matrix, which maps the control force generated by the virtual spatial spring from the virtual spring direction into the finger joint space.
In order to improve the dynamic behavior of the adaptive compliant grasp approach controller, a damping term is introduced along the direction of the spatial virtual spring between the two different fingertips. The mass matrix of the finger $\boldsymbol{M}(\boldsymbol{\theta})$ can be projected from joint space into the finger distance direction through the Jacobian $J_{1 i}$ :

$$
\boldsymbol{M}_{1 i}(\boldsymbol{\theta})=\left(\boldsymbol{J}_{1 i} \boldsymbol{M}(\boldsymbol{\theta})^{-1} \boldsymbol{J}_{1 i}^{T}\right)^{-1}
$$

Therefore the damping term $\boldsymbol{D}_{1 i}$ of the adaptive grasp controller can be achieved by the Double Diagonalization approach[20]:

$$
\boldsymbol{D}_{1 i}=\mathcal{D}\left(\boldsymbol{M}_{1 i}(\boldsymbol{\theta}), \boldsymbol{K}_{1 i}, \xi 1 i\right)
$$

where $\xi_{1 i}$ is the coefficient for the damping term design.

The adaptive compliant grasp control law based on spatial virtual spring between thumb and one of the other finger can be then written as:

$$
\boldsymbol{\tau}_{a d a}=-\boldsymbol{\tau}_{1 i}-\underbrace{\boldsymbol{J}_{1 i}^{T} \boldsymbol{D}_{1 i} \boldsymbol{J}_{1 i}}_{\boldsymbol{D}_{1 i}(\boldsymbol{\theta})} \dot{\boldsymbol{\theta}}
$$

The multi-finger adaptive compliant grasp approach controller can be deduced from the above derivation, by summing all the energy storage functions of the virtual spring framework, which can be written as:

$$
\boldsymbol{\tau}_{a d a m}=-\frac{\partial V(\boldsymbol{\theta})}{\partial \boldsymbol{\theta}}-\boldsymbol{D}(\boldsymbol{\theta}) \dot{\boldsymbol{\theta}}+g(\boldsymbol{\theta})
$$

where $\boldsymbol{\tau}_{\text {adam }}$ represents the control input vector. $\boldsymbol{D}(\boldsymbol{\theta})$ and $g(\boldsymbol{\theta})$ are the damping term and gravity term, respectively. The energy storage function of the complete virtual spatial spring system $V$ can be defined as:

$$
V=\sum_{i=2}^{n} V_{1 i}
$$

where the $V_{1 i}$ is the energy storage function of the spatial spring between the thumb and the remaining fingers. $n$ indicates the number of the fingers involved in the grasp task. Therefore the multi-finger adaptive grasp control law can be written as:

$$
\boldsymbol{\tau}_{\text {adam }}=-\sum_{i=2}^{n} \boldsymbol{\tau}_{1 i}^{n}-\sum_{i=2}^{n}\left(\boldsymbol{D}_{1 i}^{n}(\boldsymbol{\theta}) \dot{\boldsymbol{\theta}}\right)
$$

where $\tau_{\text {adam }}$ represents the actuator torque vector as control input of the multi-finger adaptive compliant control law. $\tau_{1 i}$ is described as eq. (7). With the negative joint torque feedback loop, the actual implementation of the multi-finger adaptive grasp control law can be expressed as:

$$
\boldsymbol{\tau}_{\text {grasp }}=\boldsymbol{K}_{\tau} \boldsymbol{\tau}_{\text {adam }}+\left(\boldsymbol{I}-\boldsymbol{K}_{\tau}\right) \boldsymbol{\tau}
$$

where $\tau_{\text {grasp }}$ represents the actuator torque as control input of the grasp approach controller. $\boldsymbol{\tau}$ indicates the external torque on joints of the robot finger, and $\boldsymbol{K}_{\tau}$ is a diagonal matrix representing the torque feedback gain, which contains 
$k_{\tau i} \geq 1$, determined by the noise level of the torque sensor. $\boldsymbol{K}_{\tau}$ defines the reduction ratio of the robot finger inertia reacting to external forces/torques.

\section{B. Self-collision avoidance between different fingers during the approaching phase}

During the approach phase, self-collision avoidance should be considered if the adjacent fingers are at less than the minimum safe distances. The virtual springs between adjacent fingers can be introduced so that repellent forces can be generated to prevent self-collision among digits during the grasp approaching phase, as shown in Fig. 2.

The energy storage function of the self-collision avoidance virtual springs between adjacent fingers can be written as:

$$
V_{i}^{c}= \begin{cases}\frac{1}{2} K_{i}^{c}\left(l_{i}-l_{\text {safe }}\right)^{2} & l_{i} \leq l_{\text {safe }} \\ 0 & l_{i}>l_{\text {safe }} .\end{cases}
$$

where $l_{i}=\left\|x_{i}-x_{i+1}\right\|$ indicates the distance between the $i^{t h}$ and $(i+1)^{t h}$ finger, and $l_{\text {safe }}$ represents the minimum safe distance. $K_{i}^{c}$ defines the stiffness of the self-collision avoidance virtual spring. The energy storage function of the self-collision avoidance virtual spring is similar to eq. (2), with one key difference: the control forces generated by the self-collision avoidance virtual springs are repelling forces, rather than grasping forces generated by the spatial virtual spring for the adaptive compliant grasp approach controller. The self-collision avoidance control law can be obtained by following the equations (3) (9). The final self-collision avoidance control law can be expressed as:

$$
\boldsymbol{\tau}_{c o l}=-\sum_{i=2}^{n-1} \boldsymbol{\tau}_{i}^{c}-\sum_{i=2}^{n-1} \underbrace{\boldsymbol{J}_{i}^{c T} \boldsymbol{D}_{i}^{c} \boldsymbol{J}_{i}^{c}}_{\boldsymbol{D}_{i}^{c}(\boldsymbol{\theta})} \dot{\boldsymbol{\theta}}
$$

where:

$$
\boldsymbol{\tau}_{i}^{c}= \begin{cases}-\boldsymbol{J}_{i}^{c T} K_{i}^{c}\left(l_{i}-l_{\text {safe }}\right) & l_{i} \leq l_{\text {safe }} \\ 0 & l_{i}>l_{\text {safe }}\end{cases}
$$

$\boldsymbol{J}_{i}^{c}$ represents the general Jacobian matrix, as described in eq. (7). $\tau_{c o l}$ represents the actuator torque vector as control input of the self-collision avoidance control law.

With the self-collision avoidance controller, the complete adaptive grasp control law can be expressed as:

$$
\boldsymbol{\tau}_{\text {complete }}=\boldsymbol{K}_{\tau}\left(\boldsymbol{\tau}_{\text {adam }}+\boldsymbol{\tau}_{\text {col }}\right)+\left(\boldsymbol{I}-\boldsymbol{K}_{\tau}\right) \boldsymbol{\tau}
$$

where $\boldsymbol{\tau}_{\text {complete }}$ represents the actuator torque as control input of the complete adaptive compliant grasp approach control law with self-collision avoidance between the digits. $\boldsymbol{\tau}_{\text {adam }}$ and $\boldsymbol{\tau}_{\text {col }}$ are the command joint torque vectors of the adaptive compliant grasp approach controller and the selfcollision avoidance controller, respectively. $\boldsymbol{\tau}$ and $\boldsymbol{K}_{\tau}$ are defined as eq. (14).

\section{EXPERIMENTAL RESUlts AND ANALYSis}

A. Experimental evaluation of the spatial virtual spring based adaptive compliant control

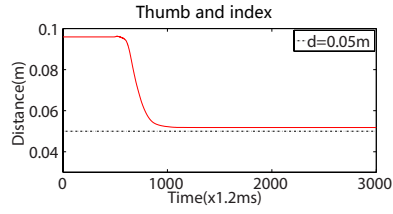

(a)

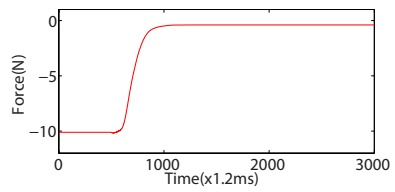

(c)

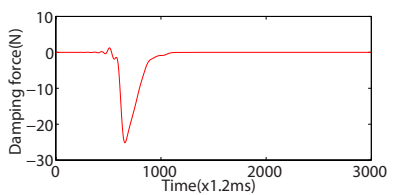

(e)

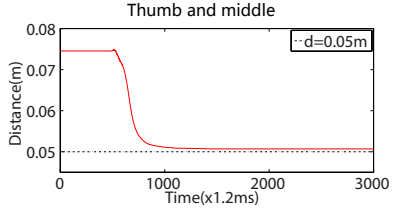

(b)

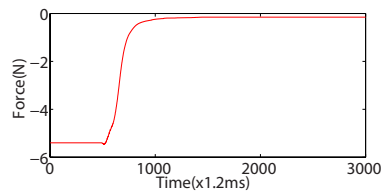

(d)

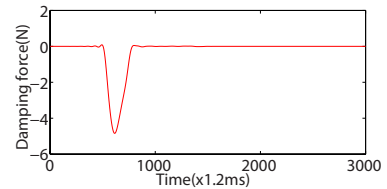

(f)
Fig. 3. Experimental results of the multi-finger adaptive compliant grasp control. (a) and (b) show the distance varying between thumb and index finger, thumb and middle finger, respectively. (c) and (d) demonstrate the spatial force generated by the spatial springs of thumb-index and thumbmiddle, with respect to the distance varying. (e) and (f) indicate the damping forces varying of the two pairs of fingers, respectively.

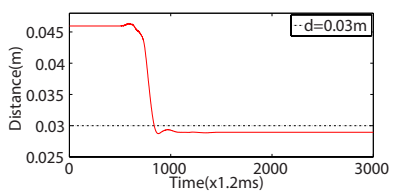

(a)

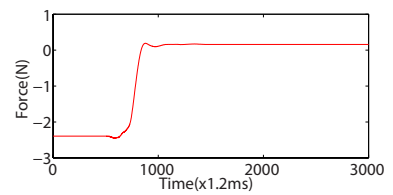

(b)

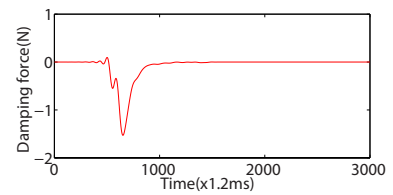

(c)

Fig. 4. Experimental results of the self-collision avoidance strategy. (a) shows the distance varying between the two fingers. (b) and (c) demonstrate repulsive force and damping response of the self-collision avoidance virtual spring with respect to the distance varying.

The experimental results of the multi-finger adaptive compliant grasp control are shown in Fig. 3. Three fingers are utilized in experiment presented here. The thumb is pushed by the operator to simulate the unexpected contact with the object. Once the contact force on the thumb reaches the threshold, the multi-finger adaptive compliant controller is triggered to complete the grasp execution task. The stiffness and damping parameters are set to be $220 \mathrm{~N} / \mathrm{m}$ and $100 \mathrm{Ns} / \mathrm{m}$, 
respectively. The experiment demonstrates that the control force increases stably with varying distance between the fingers. The thumb-index and thumb-middle finger pairs are driven by the adaptive compliant grasp controller to reach the rest length $(0.05 \mathrm{~m})$. The dynamic behavior of the controller can be improved by the damping force shown. The control error is caused by the friction and gravity forces, which can be reduced with the friction and gravity compensation methods.

The control force on the thumb is significantly higher than the remaining fingers, as it opposes four fingers. This is designed into the virtual spring configuration, shown in Fig. 2. Fig. 4 shows the results of the self-collision avoidance strategy. The stiffness and damping parameters of the selfcollision avoidance virtual spring between the index-middle virtual spring are set at $160 \mathrm{~N} / \mathrm{m}$ and $80 \mathrm{Ns} / \mathrm{m}$, respectively. The rest length of the virtual spring is chosen as $0.03 \mathrm{~m}$. Repelling force between the two fingers can be observed when the distance of the two fingers is smaller than the set rest length of the virtual spring. In other words, the fingers are driven away from each other to avoid collision.

\section{B. Grasp experiments}

In order to assess the effectiveness of the adaptive grasp control strategy, a quantitative experimental analysis is carried out by evaluating the range of errors, which can be accommodated while using adaptive grasp and non-adaptive grasp. A large number of grasps are executed, during which, all the robot fingers are commanded initially to the expected object location, as would be given by a planner. The actual position of the object are placed with various designated errors in both horizontal directions (X- and Yaxes) for the experiment trials. 10 grasp trials are conducted for every object position error settings (e.g. position $(\mathrm{x}, \mathrm{y})=(-$ $20 \mathrm{~mm}, 15 \mathrm{~mm})$ ) and finger combination ( 2-, 3- and 5-finger form). A cylindrical shaped glue stick $(\phi 30 \mathrm{~mm})$ is used as the target object in the 2-finger form grasp task and 3-finger form. A spray cleaner bottle $(\phi 50 \mathrm{~mm})$ is used in the 5finger grasp task as the target object. A trial is considered a success if the target object can be grasped and stably lifted up. More quantitative results and analysis and presented in the following section.

Fig. 5 shows the results of 2-, 3-, and 5-finger grasp experiments, which are shown as seen from point of view of above the object being grasped. The circle represents the size and surface profile of the expected position of the target object. The footprints of the fingertips in the initial configuration are shown as gray rectangle-ellipse shape. The black arrows indicates the planned grasp approach trajectories of the robot fingertips. The two axes of the chart represent the actual position of the target with respect to the expected placement.

The green region is the area where successful object grasp and lift is achieved by both adaptive grasp approach and

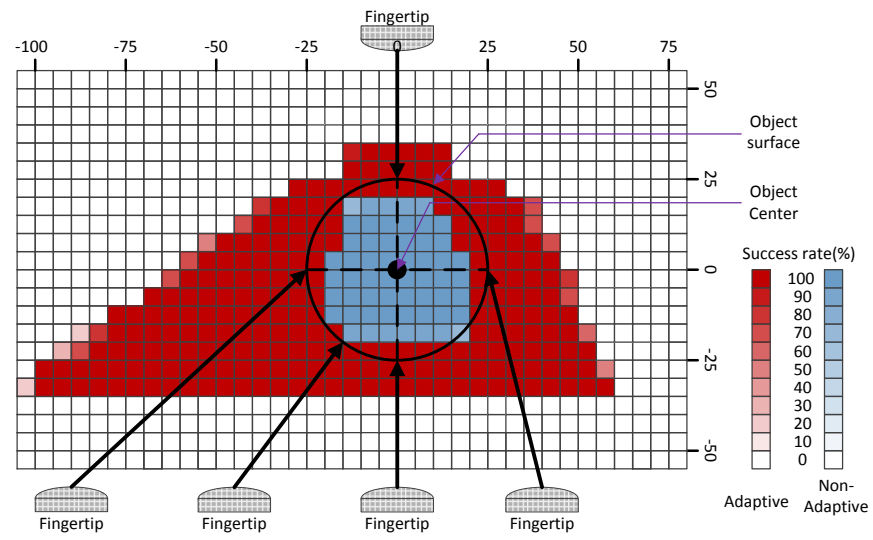

(a) 5-finger grasp

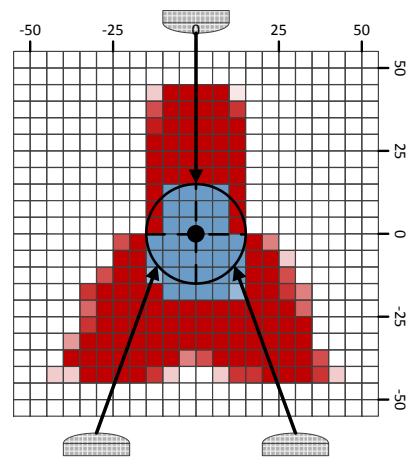

(b) 3-finger grasp

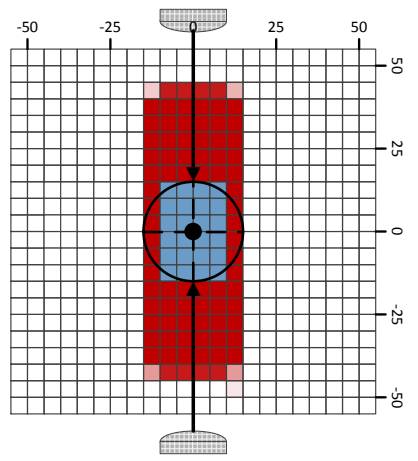

(c) 2-finger grasp
Fig. 5. Comparison of grasp success rates with adaptive compliant grasp versus non-adaptive grasp, both of which are grasping the object from the top. The green region is the area where both grasp approach strategies succeed in grasping and lifting, while the red region is the area where only adaptive grasp approach succeed. The circle represents the size and expected position of the target object. The footprints of the fingertips are shown as gray rectangle-ellipse shape. The black arrows indicates the planned grasp approach trajectories of the robot fingertips. The two axes of the chart represent the actual position of the target with respect to the expected placement.

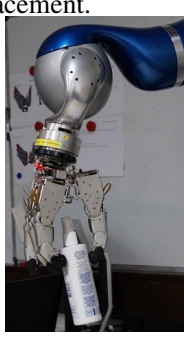

(a)

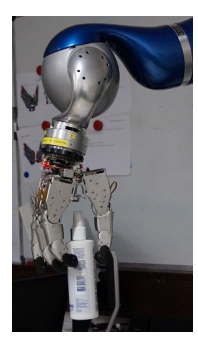

(b)

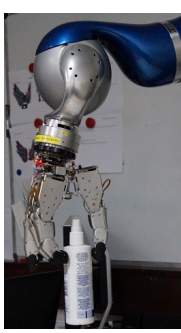

(c)

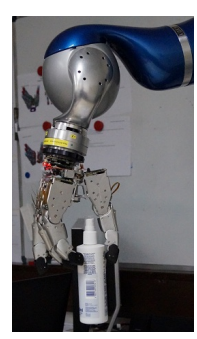

(d)
Fig. 6. The comparison of object poses after grasp accomplished with 3finger form adaptive compliant grasp versus non-adaptive grasp. (a) shows the post-grasp object pose of non-adaptive grasp with $5 \mathrm{~mm}$ displacement; (b), (c) and (d) show the post-grasp object of adaptive grasp with $5 \mathrm{~mm}$, $20 \mathrm{~mm}$, and $45 \mathrm{~mm}$ displacement.

non-adaptive grasp control. The red region is the area where adaptive grasp approach succeed in grasp and lift, while the non-adaptive grasp fails to accomplish the grasp task. The success rate is represented as the transparency rate of the 
area. It is noted that a small position error of $15 \mathrm{~mm}$ can result in failure by non-adaptive grasp in 2-finger form and 3finger form grasp. $20 \mathrm{~mm}$ position error can be accommodated in 5-finger grasp, due to the increasing finger numbers. On the other hand, the object can be displaced up to $45 \mathrm{~mm}$ in 2-finger and 3-finger grasp, and $35 \mathrm{~mm}$ in five grasp. Considering the open distance between the thumb and the remaining fingers $(120 \mathrm{~mm})$, and the size of the objects $(\phi 30 \mathrm{~mm}$ and $\phi 50 \mathrm{~mm}$ ), we observed that the position uncertainties of the object is accommodated along the planned trajectories of the fingertips, when using the adaptive grasp approach control strategy. The non-symmetrical shape of the success region during 3-finger and 5-finger grasp is a result of the non-symmetrical configuration of the fingers on the hand, where the thumb is designed to be opposing to the remaining fingers, while in closest proximity to the index finger. The size of success region rises with increasing number of fingers utilized for the grasp.

The maximum tolerable position error range is significantly enlarged with multi-finger non-adaptive grasp, which indicates that the grasp performance improves with increasing number for fingers utilized for the grasp task. Conversely as the couplings and interferences between different fingers also increases with increasing number of fingers, the adaptive compliant grasp control can also face some performance limits when a large number of fingers are utilized. In the actual implementation, the number of fingers involved in the grasp and manipulation task should be considered together with the complexity of the control strategy of the hand.

Fig. 6 shows the grasp quality comparison with 2-finger form adaptive compliant grasp versus non-adaptive grasp. Poor grasp quality can be observed even with $5 \mathrm{~mm}$ displacement, as shown in Fig. 6(a). On the other hand, good grasp quality can be achieved with $45 \mathrm{~mm}$ position error by the adaptive compliant grasp approach control strategy, as shown in Fig. 6(d).

The results of 2-finger form side grasp are shown in Fig. 7 , with adaptive grasp approach versus non-adaptive grasp approach. Up to $45 \mathrm{~mm}$ position error of the object can be accommodated by the adaptive compliant approach-to-grasp strategy, in comparison with $15 \mathrm{~mm}$ position error tolerance by the non-adaptive grasp approach. As 3- and 5-finger side grasp results are similar to 2 -finger side grasp, only 2 -finger experiment results are shown here as a representative.

Significantly larger position errors with respect to the hand workspace can be accommodated with the proposed adaptive compliant grasp control strategy. $246 \%, 391 \%$ and $333 \%$ increase in position error area coverage have been achieved in 5-finger, 3-finger, and 2-finger form overhead (approach from top) adaptive and compliant approach-tograsp strategy, respectively. 390\% increase in position error area coverage have been achieved in 2-finger side adaptive and compliant approach-to-grasp strategy. The results were

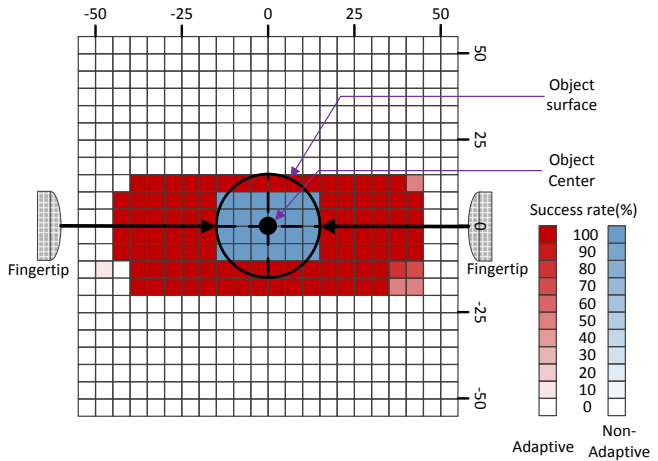

Fig. 7. The comparison of side grasp success rates with 2-finger form adaptive compliant grasp versus non-adaptive grasp

similarly successful for the 3- and 5-finger side grasps.

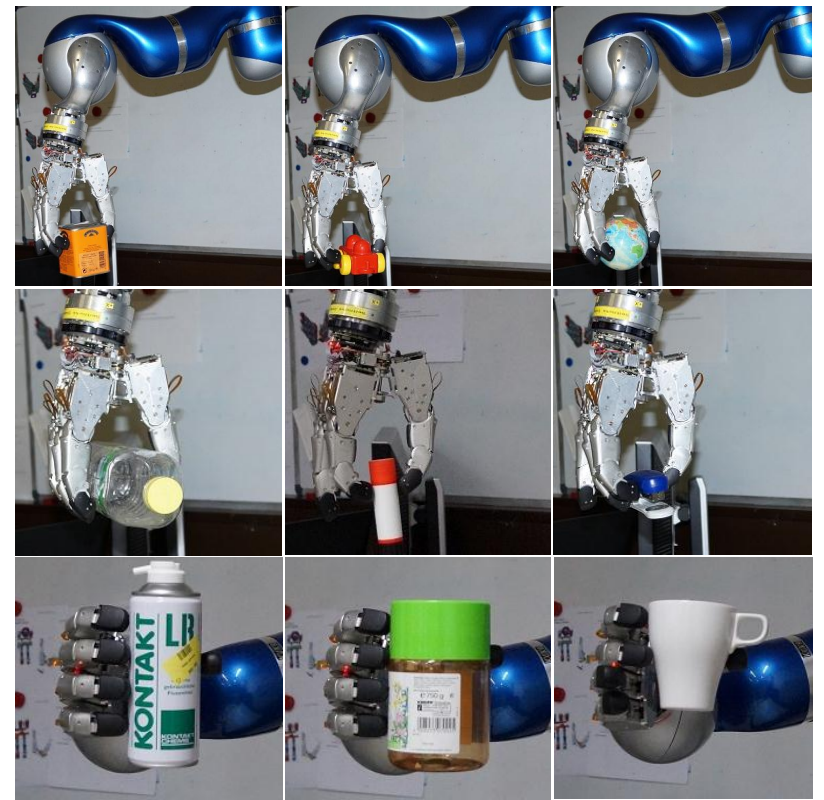

Fig. 8. Examples of successful grasps, from the side or above, of different objects with various object position errors. Thanks to the compliance behavior of the grasps, these objects can withstand some additional external disturbance, such as a light bump or a pull, and still have the grasp on the object maintained

In order to evaluate and showcase the robustness of the proposed approach-to-grasp concept, we were able to successfully grasp 12 representative dissimilarly shaped objects commonly found in daily settings (see Fig. 8). Each object is placed on a table with a randomly selected position, constrained by the workspace of the robotic hand.

231 out of 240 attempts of grasping and lifting the object are stably achieved by adaptive compliant grasp approach, in comparison with 188 successful trials by non-adaptive grasp approach. A higher success rate is demonstrated by the adaptive compliant grasp over a wide range of target objects and poses. 


\section{ADDITIONAL OBSERVATIONS}

We observed a clear advantage of the multi-finger hand during multi-finger grasp. The higher number of contact points at different position in space, afforded the multifinger dexterous hand the ability to form a finger "basket" (particularly when using more fingers) to "catch" the object after it starts moving or tipping over due to contacting the object with a position error. This "catching" style of grasping the object, often used by humans, can be quite effective given sufficient robotic finger speed. This phenomenon should be further studied. The compliant, yet disturbance tolerant, grasp behavior once the object is in stable grip, not only provides more grasp robustness, but also gives the robot operator more confidence in carrying out a task sequence. This, too, would be difficult to duplicate in a 2-jaw gripper. Finally, the full purpose of a dexterous hand is to manipulate the object after having it in its grasp. A strategy to address manipulation shall be extended into our grasp strategy pipeline going forward.

\section{CONCLUSIONS}

In order to address the object position error during grasp task, an adaptive compliant grasp control strategy is proposed in this paper. When unexpected contact is detected, the finger remains in contact with the object in a compliant manner, such that the unplanned motion of the object caused by the contact forces is minimized. At the same time, the remaining fingers not in contact with the object are adaptively driven toward the adjusted grasping position by the adaptive compliant control strategy based on a new variation on the spatial virtual spring concept. Experimental results confirm robust and consistently improved grasp performance on objects with significantly higher tolerable position error. We achieved an improvement in object position error coverage by $246 \%$ to $391 \%$ of the non-adaptive approach-to-grasp. Finally, additional qualitative observations could be made thanks to the extensive experiment trials performed for this study. It is our hope that this would help open the discussion, and pave the way to deeper understanding, on several open issues about the approach-to-grasp phases of the robot tasks and beyond.

\section{ACKNOWLEDGMENTS}

This work was partially funded by the EU project EuRoC (grant agreement no. CP-IP 608849).

\section{REFERENCES}

[1] A. T. Miller, S. Knoop, H. I. Christensen, and P. K. Allen, "Automatic grasp planning using shape primitives," in Proceedings of IEEE International Conference on Robotics and Automation, vol. 2, 2003, pp. 1824-1829.

[2] N. Y. Lii, Z. Chen, M. A. Roa, A. Maier, B. Pleintinger, and C. Borst, "Toward a task space framework for gesture commanded telemanipulation," in Proceedings of IEEE International Symposium on Robot and Human Interactive Communication. IEEE, 2012, pp. 925-932.
[3] M. Chalon, J. Reinecke, and M. Pfanne, "Online in-hand object localization," in Proceedings of IEEE International Conference on Intelligent Robots and Systems, 2013, pp. 2977-2984.

[4] T. Takahashi, T. Tsuboi, T. Kishida, Y. Kawanami, S. Shimizu, M. Iribe, T. Fukushima, and M. Fujita, "Adaptive grasping by multi fingered hand with tactile sensor based on robust force and position control," in Proceedings of IEEE International Conference on Robotics and Automation, Pasadena, CA, USA, 2008, pp. 264-271.

[5] K. Hsiao, S. Chitta, M. Ciocarlie, and E. G. Jones, "Contact-reactive grasping of objects with partial shape information," in Proceedings of IEEE International Conference on Intelligent Robots and Systems, 2010, pp. 1228-1235.

[6] S. Haddadin, M. Suppa, S. Fuchs, T. Bodenmüller, A. Albu-Schäffer, and G. Hirzinger, "Towards the robotic co-worker," in Robotics Research. Springer, 2011, pp. 261-282.

[7] N. Hogan, "Impedance control-An approach to manipulation. I-Theory. II-Implementation. III-Applications," ASME Transactions on Dynamic Systems, Measurement, and Control, vol. 107, 1985.

[8] S. Stramigioli, Modeling and IPC control of interactive mechanical systems: a coordinate-free approach. Germany: Springer, 2001, vol. 266.

[9] T. Wimböck, C. Ott, A. Albu-Schäffer, and G. Hirzinger, "Comparison of object-level grasp controllers for dynamic dexterous manipulation," The International Journal of Robotics Research, vol. 31, no. 1, pp. 3-23, 2012.

[10] E. Fasse and J. Broenink, "A spatial impedance controller for robotic manipulation," IEEE Transactions on Robotics and Automation, vol. 13 , no. 4 , pp. 546-556, 1997.

[11] O. Khatib, "Inertial properties in robotic manipulation: An object-level framework," The International Journal of Robotics Research, vol. 14, no. 1, pp. 19-36, 1995.

[12] L. Birglen, C. Gosselin, and T. Laliberté, Underactuated robotic hands. Springer, 2008, vol. 40.

[13] RobotIQ. (2010, Oct.) Robotiq adaptive gripper: Specification sheet @ ONLINE. [Online]. Available: http://www.robotiq.com

[14] A. M. Dollar and R. D. Howe, "The highly adaptive sdm hand: Design and performance evaluation," The International Journal of Robotics Research, vol. 29, no. 5, pp. 585-597, 2010.

[15] V. Tincani, M. G. Catalano, E. Farnioli, M. Garabini, G. Grioli, G. Fantoni, and A. Bicchi, "Velvet fingers: A dexterous gripper with active surfaces," in Proceedings of IEEE International Conference on Intelligent Robots and Systems, 2012, pp. 1257-1263.

[16] L. U. Odhner and A. M. Dollar, "Dexterous manipulation with underactuated elastic hands," in Proceedings of IEEE International Conference on Robotics and Automation, 2011, pp. 5254-5260.

[17] H. Liu, K. Wu, P. Meusel, N. Seitz, G. Hirzinger, M. Jin, Y. Liu, S. Fan, T. Lan, and Z. Chen, "Multisensory five-finger dexterous hand: The DLR/HIT Hand II," in Proceedings of IEEE/RSJ International Conference on Intelligent Robots and Systems, Nice, France, 2008, pp. 3692-3697.

[18] R. Bischoff, J. Kurth, G. Schreiber, R. Koeppe, A. Albu-Schäffer, A. Beyer, O. Eiberger, S. Haddadin, A. Stemmer, G. Grunwald, et al., "The kuka-dlr lightweight robot arm-a new reference platform for robotics research and manufacturing," in International symposium on Robotics, 2010, pp. 1-8.

[19] Z. Chen, N. Y. Lii, T. Wimböck, S. Fan, H. Liu, and A. Albu-Schäffer, "Experimental analysis on spatial and cartesian impedance control for the dexterous DLR/HIT II hand," International Journal of Robotics and Automation, vol. 29, no. 1, 2014.

[20] A. Albu-Schäffer, C. Ott, and G. Hirzinger, "A passivity based Cartesian impedance controller for flexible joint robots-Part II: full state feedback, impedance design and experiments," in Proceedings of IEEE International Conference on Robotics and Automation, New Orleans, LA, USA, 2004, pp. 2666-2672. 